International Journal of Pure and Applied Mathematics

Volume 88 No. 2 2013, 263-272

ISSN: 1311-8080 (printed version); ISSN: 1314-3395 (on-line version)

url: http://www.ijpam.eu

doi: http://dx.doi.org/10.12732/ijpam.v88i2.9

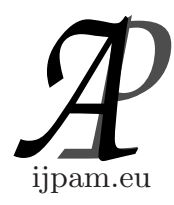

\title{
ON A HYPERSURFACE OF \\ A HYPERBOLIC COSINE FINSLER METRIC
}

\author{
H. Wosoughi \\ Department of Mathematics \\ Islamic Azad University \\ Babol Branch, IRAN
}

\begin{abstract}
The purpose of the present paper is to investigate the various kinds of hypersurface of Finsler space with $(\alpha, \beta)$ - metric of type $L=\alpha \cosh \left(\frac{\beta}{\alpha}\right)$. The conditions under which this hypersuface to be a hyperplane of first, second or third kinds have been obtained.
\end{abstract}

AMS Subject Classification: 53B40, 53C60

Key Words: Finsler space, hypersurface, induced Cartan connection, $(\alpha, \beta)$ metric, hyperplane of $1^{\text {st }}, 2^{\text {nd }}$ and $3^{\text {rd }}$ kinds

\section{Introduction}

Let $F^{n}=\left(M^{n}, L\right)$ be an n-dimensional Finsler space,i.e., a pair consisting of an n-dimensional differential manifold $M^{n}$ equipped with a fundamental function $\mathrm{L}(x, y)$. The concept of the $(\alpha, \beta)$-metric was introduced by M. Matsumoto [4] and has been studied by many authors (see [1], [2], [7],..), where $\alpha^{2}=$ $a_{i j}(x) y^{i} y^{j}$ is a Riemannian metric and $\beta=b_{i}(x) y^{i}$ is a 1 -form on $M^{n}$.

A hypersurface $M^{n-1}$ of the $M^{n}$ may be represented parametrically by the equation $x^{i}=x^{i}\left(u^{\alpha}\right), \alpha=1, \ldots, n-1$, where $u^{\alpha}$ are Gaussian coordinates on $M^{n-1}$.

Received: July 13, 2013

(c) 2013 Academic Publications, Ltd. url: www.acadpubl.eu 
Since the function $\underline{L}=: L(x(u), y(u, v))$ gives rise to a Finsler metric of $M^{n-1}$, we get an (n-1)-dimensional Finsler space $F^{n-1}=\left(M^{n-1}, \underline{L}(u, v)\right)$. The hypersurface of Finler Space with some given special metrics has been studied by authors (see [8], [9], [10]).

with $(\alpha, \beta)$-metric $L=\alpha \cosh \left(\frac{\beta}{\alpha}\right)$ and the hypersurface of $F^{n}$ with $b_{i}=\partial_{i} b$ being the

In the present paper, we consider an n-dimensional Finsler space $F^{n}=$ $\left(M^{n}, L\right)$ gradient of a scalar function $b(x)$. We prove the conditions for this hypersurface to be hyperplane of $1^{\text {st }}$ kind, $2^{\text {nd }}$ kind and $3^{\text {rd }}$ kind.

\section{Preliminaries}

Let $F^{n}=\left(M^{n}, L\right)$ be a special Finsler space with the metric

$$
L(\alpha, \beta)=\alpha \cosh \left(\frac{\beta}{\alpha}\right)
$$

The derivatives of the (1) with respect to $\alpha$ and $\beta$ are given by

$$
\begin{aligned}
& L_{\alpha}=\cosh \left(\frac{\beta}{\alpha}\right)-\frac{\beta}{\alpha} \sinh \left(\frac{\beta}{\alpha}\right), \\
& L_{\beta}=\sinh \left(\frac{\beta}{\alpha}\right), \\
& L_{\alpha \alpha}=\frac{\beta^{2}}{\alpha^{3}} \cosh \left(\frac{\beta}{\alpha}\right), \\
& L_{\beta \beta}=\frac{1}{\alpha} \cosh \left(\frac{\beta}{\alpha}\right), \\
& L_{\alpha \beta}=-\frac{\beta}{\alpha^{2}} \cosh \left(\frac{\beta}{\alpha}\right),
\end{aligned}
$$

where $L_{\alpha}=\partial L / \partial \alpha, L_{\beta}=\partial L / \partial \beta, L_{\alpha \alpha}=\partial L_{\alpha} / \partial \alpha, L_{\beta \beta}=\partial L_{\beta} / \partial \beta$ and $L_{\alpha \beta}=$ $\partial L_{\alpha} / \partial \beta$.

In the special Finsler space $F^{n}=\left(M^{n}, L\right)$ the normalized element of support $l_{i}=\dot{\partial} L$ and the angular metric tensor $h_{i j}$ are given by [6]:

$$
\begin{aligned}
& l_{i}=\alpha^{-1} L_{\alpha} Y_{i}+L_{\beta} b_{i}, \\
& h_{i j}=p a_{i j}+q_{0} b_{i} b_{j}+q_{1}\left(b_{i} Y_{j}+b_{j} Y_{i}\right)+q_{2} Y_{i} Y_{j}
\end{aligned}
$$

where

$$
\begin{aligned}
& Y_{i}=a_{i j} y^{j} \\
& p=L L_{\alpha} \alpha^{-1}=\frac{1}{\alpha}\left(\cosh \left(\frac{\beta}{\alpha}\right)\right)\left(\alpha \cosh \left(\frac{\beta}{\alpha}\right)-\beta \sinh \left(\frac{\beta}{\alpha}\right),\right. \\
& q_{0}=L L_{\beta \beta}=\cosh ^{2}\left(\frac{\beta}{\alpha}\right) \\
& q_{1}=L L_{\alpha \beta} \alpha^{-1}=-\frac{\beta}{\alpha^{2}} \cosh ^{2}\left(\frac{\beta}{\alpha}\right) \\
& q_{2}=L \alpha^{-2}\left(L_{\alpha \alpha}-L_{\alpha} \alpha^{-1}\right)=\frac{1}{\alpha^{4}}\left(\cosh \left(\frac{\beta}{\alpha}\right)\right)\left[\left(\beta^{2}-\alpha^{2}\right) \cosh \left(\frac{\beta}{\alpha}\right)+\alpha \beta \sinh \left(\frac{\beta}{\alpha}\right)\right]
\end{aligned}
$$


The fundamental tensor $g_{i j}=\frac{1}{2} \dot{\partial} \dot{\partial} L^{2}$ and it's reciprocal tensor is given by [6]

$$
g_{i j}=p a_{i j}+p_{0} b_{i} b_{j}+p_{1}\left(b_{i} Y_{j}+b_{j} Y_{i}\right)+p_{2} Y_{i} Y_{j},
$$

where

$$
\begin{gathered}
p_{0}=q_{0}+L_{\beta}^{2}=\cosh 2(\beta / \alpha) \\
p_{1}=q_{1}+L^{-1} p L_{\beta}=\frac{1}{\alpha^{2}}\left\{-\beta \cosh ^{2}\left(\frac{\beta}{\alpha}\right)+\sinh \left(\frac{\beta}{\alpha}\right)\left[\alpha \cosh \left(\frac{\beta}{\alpha}\right)-\beta \sinh \left(\frac{\beta}{\alpha}\right)\right]\right\} \\
p_{2}=q_{2}+p^{2} L^{-2}=\frac{1}{\alpha^{4}}\left\{\cosh \left(\frac{\beta}{\alpha}\right)\left[\left(\beta^{2}-\alpha^{2}\right) \cosh \left(\frac{\beta}{\alpha}\right)+\alpha \beta \sinh \left(\frac{\beta}{\alpha}\right)\right]+\right. \\
\left.\left[\alpha \cosh \left(\frac{\beta}{\alpha}\right)-\beta \sinh \left(\frac{\beta}{\alpha}\right)\right]^{2}\right\} \\
g^{i j}=p^{-1} a^{i j}+S_{0} b^{i} b^{j}+S_{1}\left(b^{i} y^{j}+b^{j} y^{i}\right)+S_{2} y^{i} y^{j},
\end{gathered}
$$

where

$$
\begin{aligned}
& b^{i}=a^{i j} b_{j}, \\
& S_{0}=\left(p p_{0}+\left(p_{0} p_{2}-p_{1}^{2}\right) \alpha^{2}\right) / \varsigma \\
& S_{1}=\left(p p_{1}+\left(p_{0} p_{2}-p_{1}^{2}\right) \beta\right) / \varsigma p \\
& S_{2}=\left(p p_{2}+\left(p_{0} p_{2}-p_{1}^{2}\right) b^{2}\right) / \varsigma p \\
& b^{2}=a_{i j} b^{i} b^{j} \\
& \varsigma=p\left(p+p_{0} b^{2}+p_{1} \beta\right)+\left(p_{0} p_{2}-p_{1}^{2}\right)\left(\alpha^{2} b^{2}-\beta^{2}\right)
\end{aligned}
$$

The $h v$-torsion tensor $C_{i j k}=\frac{1}{2} \dot{\partial} g_{i j}$ is given by [6]

$$
2 p C_{i j k}=p_{1}\left(h_{i j} m_{k}+h_{j k} m_{i}+h_{k i} m_{j}\right)+\gamma_{1} m_{i} m_{j} m_{k},
$$

where

$$
\gamma_{1}=p \frac{\partial p_{0}}{\partial \beta}-3 p_{1} q_{0}, \quad m_{i}=b_{i}-\alpha^{-2} \beta Y_{i}
$$

Here $m_{i}$ be a non-vanishing covariant vector orthogonal to the element of support $y^{i}$.

Let $\left\{\begin{array}{l}i \\ j k\end{array}\right\}$ be the component of Christoffel symbols of the associated Riemannian space $R^{n}$ and $\nabla_{k}$ be covariant differentiation with respect to $x^{k}$ relative to this Christoffel symbols. We put

$$
2 E_{i j}=b_{i j}+b_{i j}, \quad 2 F_{i j}=b_{i j}-b_{j i}
$$

where $b_{i j}=\nabla_{j} b_{i}$.

Let $\mathrm{C} \Gamma=\left(\Gamma_{j k}^{* i}, \Gamma_{0 k}^{* i}, C_{j k}^{i}\right)$ be the Cartan connection of $F^{n}$. The difference tensor $D_{j k}^{i}=\Gamma_{j k}^{* i}-\left\{\begin{array}{c}i \\ j k\end{array}\right\}$ of the special Finsler space $F^{n}$ is given by [3]

$$
\begin{aligned}
D_{j k}^{i}= & B^{i} E_{j k}+F_{k}^{i} B_{j}+F_{j}^{i} B_{k}+B_{j}^{i} b_{0 k}+B_{k}^{i} b_{0 j} \\
& -b_{0 m} g^{i m} B_{j k}-C_{j m}^{i} A_{k}^{m}-C_{k m}^{i} A_{j}^{m}+C_{j k m} A_{s}^{m} g^{i s} \\
& +\lambda^{s}\left(C_{j m}^{i} C_{s k}^{m}+C_{k m}^{i} C_{s j}^{m}-C_{j k}^{m} C_{m s}^{i}\right),
\end{aligned}
$$


where

$$
\begin{aligned}
& B_{k}=p_{0} b_{k}+p_{1} Y_{k}, \quad B^{i}=g^{i j} B_{j}, \quad F_{i}^{k}=g^{k j} F_{j i} \\
& B_{i j}=\left\{p_{1}\left(a_{i j}-\alpha^{-2} Y_{i} Y_{j}\right)+\frac{\partial p_{0}}{\partial \beta} m_{i} m_{j}\right\} / 2 \\
& B_{i}^{k}=g^{k j} B_{j i} \\
& A_{k}^{m}=b_{k}^{m} E_{00}+B^{m} E_{k 0}+B_{k} F_{0}^{m}+B_{0} F_{k}^{m} \\
& \lambda^{m}=B^{m} E_{00}+2 B_{0} F_{0}^{m}, \quad B_{0}=B_{i} y^{i}
\end{aligned}
$$

where ' 0 ' denote contraction with $y^{i}$ except for the quantities $p_{0}, q_{0}$ and $S_{0}$.

\section{Induced Cartan Connection}

Let $F^{n-1}$ be a hypersurface of $F^{n}$ given by the equations $x^{i}=x^{i}\left(u^{\alpha}\right)$. The element of support $y^{i}$ of $F^{n}$ is to be taken tangential to $F^{n-1}$, that is

$$
y^{i}=B_{\alpha}^{i}(u) v^{\alpha} .
$$

The metric tensor $g_{\alpha \beta}$ and $v$-torsion tenor $C_{\alpha \beta \gamma}$ of $F^{n-1}$ are given by

$$
g_{\alpha \beta}=g_{i j} B_{\alpha}^{i} B_{\beta}^{j}, \quad C_{\alpha \beta \gamma}=C_{i j k} B_{\alpha}^{i} B_{\beta}^{j} B_{\gamma}^{k}
$$

At each point $u^{\alpha}$ of $F^{n-1}$, a unit normal vector $N^{i}(u, v)$ is defined by

$$
g_{i j}(x(u, v), y(u, v)) B_{\alpha}^{i} N^{j}=0, \quad g_{i j}(x(u, v), y(u, v)) N^{i} N^{j}=1 .
$$

As for the angular metric tensor $h_{i j}$, we have

$$
h_{\alpha \beta}=h_{i j} B_{\alpha}^{i} B_{\beta}^{j}, h_{i j} B_{\alpha}^{i} N^{j}=0, h_{i j} N^{i} N^{j}=1
$$

If $\left(B_{i}^{\alpha}, N_{i}\right)$ denote the inverse of $\left(B_{\alpha}^{i}, N^{i}\right)$, then we have

$$
\begin{aligned}
& B_{i}^{\alpha}=g^{\alpha \beta} g_{i j} B_{\beta}^{j}, \quad B_{\alpha}^{i} B_{i}^{\beta}=\delta_{\alpha}^{\beta}, \\
& B_{i}^{\alpha} N^{i}=0, \quad B_{\alpha}^{i} N_{i}=0, \quad N_{i}=g_{i j} N^{i}, \\
& B_{i}^{k}=g^{k j} B_{j i}, \\
& B_{\alpha}^{i} B_{j}^{\alpha}+N^{i} N_{j}=\delta_{j}^{i}
\end{aligned}
$$

The induced connection $I C \Gamma=\left(\Gamma_{\beta \gamma}^{* \alpha}, G_{\beta}^{\alpha}, C_{\beta \gamma}^{\alpha}\right)$ of

$$
\begin{aligned}
& \Gamma_{\beta \gamma}^{* \alpha}=B_{i}^{\alpha}\left(B_{\beta \gamma}^{i}+\Gamma_{j k}^{* i} B_{\beta}^{j} B_{\gamma}^{k}\right)+M_{\beta}^{\alpha} H_{\gamma}, \\
& G_{\beta}^{\alpha}=B_{i}^{\alpha}\left(B_{0 \beta}^{i}+\Gamma_{0 j}^{* i} B_{\beta}^{j}\right), \\
& G_{\beta \gamma}^{\alpha}=B_{i}^{\alpha} C_{j k}^{i} B_{\beta}^{j} B_{\gamma}^{k},
\end{aligned}
$$


where

$$
M_{\beta \gamma}=N_{i} C_{j k}^{i} B_{\beta}^{j} B_{\gamma}^{k}, \quad M_{\beta}^{\alpha}=g^{\alpha \gamma} M_{\beta \gamma}, H_{\beta}=N_{i}\left(B_{0 \beta}^{i}+\Gamma_{0 j}^{* i} B_{\beta}^{j}\right)
$$

and $B_{\beta \gamma}^{i}=\partial B_{\beta}^{i} / \partial u^{\gamma}, B_{0 \beta}^{i}=B_{\alpha \beta}^{i} v^{\alpha}$. The quantities $M_{\beta \gamma}$ and $H_{\beta}$ are called the second fundamental $v$-tensor and normal curvature vector respectively [5]. The second fundamental $h$-tensor $H_{\beta \gamma}$ is defined as [5]

$$
H_{\beta \gamma}=N_{i}\left(B_{\beta \gamma}^{i}+\Gamma_{j k}^{* i} B_{\beta}^{j} B_{\gamma}^{k}\right)+M_{\beta} H_{\gamma}
$$

where

$$
M_{\beta}=N_{i} C_{j k}^{i} B_{\beta}^{j} N^{k} .
$$

The relative $h$ and $v$-covariant derivatives of projection factor $B_{\alpha}^{i}$ with respect to $I C \Gamma$ are given by

$$
B_{\alpha \mid \beta}^{i}=H_{\alpha \beta} N^{i},\left.B_{\alpha}^{i}\right|_{\beta}=M_{\alpha \beta} N^{i} .
$$

The equation (13) shows that $H_{\beta \gamma}$ is generally not symmetric and

$$
H_{\beta \gamma}-H_{\gamma \beta}=M_{\beta} H_{\gamma}-M_{\gamma} H_{\beta} .
$$

The above equation yield

$$
H_{0 \gamma}=H_{\gamma}, H_{\gamma 0}=H_{\gamma}+M_{\gamma} H_{0} .
$$

We use following lemmas which are due to Matsumoto [5]:

Lemma 1. The normal curvature $H_{0}=H_{\beta} v^{\beta}$ vanishes if and only if the normal curvature vector $H_{\beta}$ vanishes.

Lemma 2. A hypersurface $F^{n-1}$ is a hyperplane of $1^{\text {st }}$ kind if and only if $H_{\alpha}=0$.

Lemma 3. A hypersurface $F^{n-1}$ is a hyperplane of the $2^{\text {nd }}$ kind with respect to the connection $C \Gamma$ if and only if $H_{\alpha}=0$. and $H_{\alpha \beta}=0$.

Lemma 4. A hyperplane of the $3^{r d}$ kind is characterized by $H_{\alpha \beta}=0$ and $M_{\alpha \beta}=0$. 


\section{Hypersurface $F^{n-1}(c)$ of the Special Finsler Space}

Let us consider special Finsler metric $L=\alpha \cosh \left(\frac{\beta}{\alpha}\right)$ with a gradient $b_{i}(x)=\partial_{i} b$ for a scalar function $b(x)$ and a hypersurface $F^{n-1}(c)$ given by the equation $b(x)=\mathrm{c}\left(\right.$ constant) (see [8], [10]). From parametric equation $x^{i}=x^{i}\left(u^{\alpha}\right)$ of $F^{n-1}(c)$, we get $\partial_{\alpha} b(x(u))=0=b_{i} B_{\alpha}^{i}$, so that $b_{i}(x)$ are regarded as covariant components of a normal vector field of $F^{n-1}(c)$. Therefore, along the $F^{n-1}(c)$. Therefore, along the $F^{n-1}(c)$ we have

$$
b_{i} B_{\alpha}^{i}=0, \quad b_{i} y^{i}=0
$$

The induced metric $L(u, v)$ of $F^{n-1}(c)$ is given by

$$
L(u, v)=a_{i j} v^{\alpha} v^{\beta}, \quad a_{\alpha \beta}=a_{i j} B_{\alpha}^{i} B_{\beta}^{j},
$$

which is the Riemannian metric. At a point of $F^{n-1}(c)$, from (2), (3) and (5), we have

$$
\begin{aligned}
& p=1, \quad q_{0}=1, \quad q_{2}=-\alpha^{-2}, \quad p_{0}=1, \quad p_{1}=0, \quad p_{2}=0, \\
& \varsigma=1+b^{2}, \quad S_{0}=\frac{1}{1+b^{2}}, \quad S_{1}=0, \quad S_{2}=0 .
\end{aligned}
$$

Therefore, from (4) we get

$$
g^{i j}=a^{i j}-\frac{1}{1+b^{2}} b^{i} b^{j} .
$$

Thus along $F^{n-1}(c),(21)$ and (18) lead to $g^{i j} b_{i} b_{j}=\frac{b^{2}}{1+b^{2}}$. Therefore, we get

$$
b_{i}(x(u))=\sqrt{\frac{b^{2}}{1+b^{2}}} N_{i}, b^{2}=a^{i j} b_{i} b_{j},
$$

where $\mathrm{b}$ is the length of the vector $b^{i}$. Again from (21) and (22) we get

$$
b^{i}=a^{i j} b_{j}=\sqrt{b^{2}\left(1+b^{2}\right)} N^{i}+b^{2} \alpha^{-1} y^{i} .
$$

Thus we have

Theorem 4.1. In the special Finsler hypersurface $F^{n-1}(c)$, the induced metric a is Riemannian metric given by (19) and the scalar function $b(x)$ is given by (22) and (23).

Theorem 4.2. The second fundamental $v$-tensor of special Finsler hypersurface $F^{n-1}(c)$ vanishes and the second fundamental $h$-tensor $H_{\alpha \beta}$ is symmetric. 
Proof. The angular metric tensor and metric tensor of $F^{n}$ are given by

$$
h_{i j}=a_{i j}+b_{i} b_{j}-\frac{Y_{i} Y_{j}}{\alpha^{2}}, g_{i j}=a_{i j}+b_{i} b_{j}
$$

From (18), (24) and (11) it follows that if $h_{\alpha \beta}^{(a)}$ denote the angular metric tensor of the Riemannian $a_{i j}(x)$, then along $F^{n-1}(c), h_{\alpha \beta}=h_{\alpha \beta}^{(a)}$. From (3), we get

$$
\frac{\partial p_{0}}{\partial \beta}=\left(\frac{2}{\alpha}\right) \sinh 2\left(\frac{\beta}{\alpha}\right) .
$$

Thus along $F^{n-1}(c), \frac{\partial p_{0}}{\partial \beta}=0$ and therefore gives $\gamma_{1}=0, m_{i}=b_{i}$. Therefore the $h v$-torsion tensor becomes

$$
C_{i j k}=0
$$

in a special Finsler hypersurface $F^{n-1}(c)$. Therefore, (11), (21), (14), (18) and (25) give

$$
M_{\alpha \beta}=0, M_{\alpha}=0 \text {. }
$$

Now (16) implies that $H_{\alpha \beta}$ is symmetric.

Next, we give conditions under which $F^{n-1}(c)$ is a hyperplane of first, second and third kind:

Theorem 4.3. The hypersurface $F^{n-1}(c)$ of special Finsler space is hyperplane of first kind if and only if $2 b_{i j}=b_{i} c_{j}+b_{j} c_{i}$ holds.

Proof. From (18), we obtain $b_{i \mid j} B_{\alpha}^{i}+b_{i} B_{\alpha \mid \beta}^{i}=0$. Thus, from (15) and using $b_{i \mid j}=b_{i \mid j} B_{\beta}^{j}+\left.b_{i}\right|_{j} N^{i} H_{\beta}$, we get

$$
b_{i \mid j} B_{\alpha}^{i} B_{\beta}^{j}+\left.b_{i}\right|_{j} B_{\alpha}^{i} N^{j} H_{\beta}+b_{i} H_{\alpha \beta} N^{i}=0 .
$$

Since $\left.b_{i}\right|_{j}=-b_{h} C_{i j}^{h}$, we get

$$
\left.b_{i}\right|_{j} B_{\alpha}^{i} N^{j}=0
$$

Thus (27) gives

$$
\sqrt{\frac{b^{2}}{1+b^{2}}} H_{\alpha \beta}+b_{i \mid j} B_{\alpha}^{i} B_{\beta}^{j}=0 .
$$

It is noted that $b_{i \mid j}$ is symmetric. Furthermore, contracting (28) with $v^{\beta}$ and then with $v^{\alpha}$ and using (10), (17) and (26) we get

$$
\sqrt{\frac{b^{2}}{1+b^{2}}} H_{\alpha}+b_{i \mid j} B_{\alpha}^{i} y^{j}=0
$$




$$
\sqrt{\frac{b^{2}}{1+b^{2}}} H_{0}+b_{i \mid j} y^{i} y^{j}=0 .
$$

In view of lemmas (1) and (2), the hypersurface $F^{n-1}(c)$ is hyperplane of the first kind if and only if $H_{0}=0$. Thus from (30) it follows that $F^{n-1}(c)$ is a hyperplane of the first kind if and only if $b_{i \mid j} y^{i} y^{j}=0$. Here $b_{i \mid j}$ being the covariant derivative with respect to $C \Gamma$ of $F^{n}$ depends on $y^{i}$.

Since $b_{i}$ is a gradient vector, from (7) we have $E_{i j}=b_{i j}, F_{i j}=0$ and $F_{j}^{i}=0$. Thus (8) reduces to

$$
\begin{aligned}
& D_{j k}^{i}=B^{i} b_{j k}+B_{j}^{i} b_{0 k}+B_{k}^{i} b_{0 j}-b_{0 m} g^{i m} B_{j k} \\
&-C_{j m}^{i} A_{k}^{m}-C_{k m}^{i} A_{j}^{m}+C_{j k m} A_{s}^{m} g^{i s} \\
&+\lambda^{n}\left(C_{j m}^{i} C_{s k}^{m}+C_{k m}^{i} C_{s j}^{m}-C_{j k}^{m} C_{m s}^{i}\right) .
\end{aligned}
$$

In view of (20) and (21), the relations in (9) become to

$$
\begin{aligned}
& B_{i}=b_{i}, \quad B^{i}=\frac{b^{i}}{1+b^{2}} \\
& B_{i j}=0 \\
& B_{j}^{i}=g^{i l} B_{l j}=0 \\
& A_{k}^{m}=B^{m} b_{k 0}, \quad \lambda^{m}=B^{m} b_{00} .
\end{aligned}
$$

By virtue of (32) we have $B_{0}^{i}=0, B_{i 0}=0$ which leads $A_{0}^{m}=B^{m} b_{00}$. Therefore we have

$$
\begin{gathered}
D_{j 0}^{i}=B^{i} b_{j 0}, \\
D_{00}^{i}=B^{i} b_{00}=\left(\frac{b^{i}}{1+b^{2}}\right) b_{00} .
\end{gathered}
$$

Thus from the relation (18), we get

$$
\begin{aligned}
& b_{i} D_{j 0}^{i}=\frac{b^{2}}{1+b^{2}} b_{j 0}, \\
& b_{i} D_{00}^{i}=\frac{b^{2}}{1+b^{2}} b_{00} .
\end{aligned}
$$

From (25) it follows that

$$
b^{m} b_{i} C_{j m}^{i} B_{\alpha}^{j}=b^{2} M_{\alpha}=0 .
$$

Therefore, the relation $b_{i \mid j}=b_{i j}-b_{r} D_{i j}^{r}$ and equations (35), (36) give

$$
b_{i \mid j} y^{i} y^{j}=b_{00}-b_{r} D_{00}^{r}=\left(\frac{1}{1+b^{2}}\right) b_{00}
$$


Consequently, (29) and (30) may be written as

$$
\begin{gathered}
\sqrt{b^{2}} H_{\alpha}+\left(\frac{1}{\sqrt{1+b^{2}}}\right) b_{i 0} B_{\alpha}^{i}=0 \\
\sqrt{b^{2}} H_{0}+\left(\frac{1}{\sqrt{1+b^{2}}}\right) b_{00}=0 .
\end{gathered}
$$

Thus the condition $H_{0}=0$ is equivalent to $b_{00}=0$, where $b_{i j}$ does not depend on $y^{i}$. Since $y^{i}$ is to satisfy (18), the condition is written as $b_{i j}=\left(b_{i} y^{i}\right)\left(c_{j} y^{j}\right)$ for some $c_{j}(x)$, so that we have

$$
2 b_{i j}=b_{i} c_{j}+b_{j} c_{i},
$$

which completes the proof of the theorem.

Theorem 4.4. If the special hypersurface $F^{n-1}(c)$ of Finsler space is a hyperplane of the first kind then it is a hyperplane of the second kind, too.

Proof. using (25), (31) and (32), we get $b_{k} D_{i j}^{k}=\frac{b^{2}}{1+b^{2}} b_{i j}$. Substituting (39) in (28) and applying (18), we obtain

$$
H_{\alpha \beta}=0
$$

Therefore, by the help of Lemmas (1), (2) and (3) and Theorem (4.3) we get the required result.

Theorem 4.5. The hypersurface $F^{n-1}(c)$ of Finsler space is a hyperplane of third kind if and only if it is a hyperplane of the first kind.

Proof. This follows from (17), (40) and Theorem 4.2.

\section{References}

[1] M. Hashiguchi, Y. Ichijyo, On some special $(\alpha, \beta)$-metric, Rep. Fac. Sci. Kagasima Univ., 8 (1975), 39-46.

[2] S. Kikuchi, On the condition that a space with $(\alpha, \beta)$-metric be locally Minkowskian, Tensor, N.S., 33 (1979), 242-246.

[3] M. Matsumoto, Foundations of Finsler Geometry and Special Finsler Spaces, Kaiseisha press, Saikawa, Otsu, Japan (1986).

[4] M. Matsumoto, Theory of Finsler spaces with $(\alpha, \beta)$-metric, Rep. Math. Phys., 30 (1991). 
[5] M. Matsumoto, The induced and intrinsic Finsler connection of a hypersurface and Finslerian projective geometry, J. Math. Kyoto Univ., 25 (1985), 107-144.

[6] G. Randers, On an asymmetrical metric in the four-space of general relativity, Phys. Rev., 59, No. 2 (1941), 195-199.

[7] C. Shibata, On Finsler spaces with an $(\alpha, \beta)$-metric, J. Hokkaido Univ. of Education, IIA, 35 (1984), 1-16.

[8] U.P. Singh, Bindu Kumari, On a hypersurface of a Matsumoto space, Indian J. Pure Appl. Math., 32, No. 4 (2001), 521-531.

[9] H. Wosoughi, On a hypersuface of special Finsler space with an exponential $(\alpha, \beta)$-metric, International Journal of Contemporary Mathematical Sciences, 6, No. 4 (2011), 1969-1980.

[10] I.L. Yong Lee, Ha-Yong Park, Yong-Duk Lee, On a hypersuface of a special Finsler space with a metric $\alpha+\beta^{2} / \alpha$, Korian J. Math. Sciences, 8, No. 1 (2001), 93-101. 